\title{
miR-20a induces cisplatin resistance of a human gastric cancer cell line via targeting CYLD
}

\author{
MINGXIA ZHU ${ }^{1 *}$, XIN ZHOU $^{1 *}$, YIPING DU ${ }^{2}$, ZEBO HUANG $^{1}$, JUN ZHU $^{3}$, JING XU $^{1}$, GONGMING CHENG $^{4}$, \\ YONGQIAN SHU ${ }^{1}$, PING LIU ${ }^{1}$, WEI ZHU ${ }^{1}$ and TONGSHAN WANG ${ }^{1}$ \\ ${ }^{1}$ Department of Oncology, First Affiliated Hospital of Nanjing Medical University, Nanjing, Jiangsu 210029; \\ ${ }^{2}$ Department of Oncology, The First People's Hospital of Kunshan Affiliated With Jiangsu University, Suzhou, Jiangsu 215300; \\ ${ }^{3}$ Department of Radiation Oncology, Jiangsu Cancer Hospital, Nanjing, Jiangsu 210009; ${ }^{4}$ Department of General Surgery, \\ First Affiliated Hospital of Nanjing Medical University, Nanjing, Jiangsu 210029, P.R. China
}

Received July 23, 2015; Accepted June 1, 2016

DOI: $10.3892 / \mathrm{mmr} .2016 .5413$

\begin{abstract}
The dysregulation of microRNAs (miRNAs) has been demonstrated to contribute to drug resistance of cancer cells, and sustained nuclear factor $(\mathrm{NF}) \kappa \mathrm{B}$ activation is also pivotal in tumor resistance to chemotherapy. In the present study, an essential role for miRNA (miR)-20a was identified in the regulation of gastric cancer (GC) chemoresistance. The expression level of miR-20a was assayed by reverse transcription-quantitative polymerase chain reaction. Additionally, 3-(4,5-dimethylthiazol-2-yl)-2,5-diphenyltetrazolium bromide was used to detect the drug-resistance phenotype changes of cancer cells associated with upregulation or downregulation of miR-20a. Protein expression levelss were measured by western blotting and immunohistochemistry. Flow cytometry was used to detect cisplatin-induced apoptosis. It was found that miR-20a was markedly upregulated in GC plasma and tissue samples. Additionally, miR-20a was upregulated in GC plasma and tissues from patients with cisplatin (DDP) resistance, and in the DPP-resistant gastric cancer cell line (SGC7901/DDP). The expression of miR-20a was inversely correlated with the expression of cylindromatosis (CYLD). Subsequently, the assessment of luciferase activity verified that CYLD was a direct target gene of miR-20a. Treatment with miR-20a inhibitor increased the protein expression of
\end{abstract}

Correspondence to: Dr Wei Zhu or Dr Tongshan Wang, Department of Oncology, First Affiliated Hospital of Nanjing Medical University, 300 Guangzhou Road, Nanjing, Jiangsu 210029, P.R. China

E-mail: zhuwei@njmu.edu.cn

E-mail: kingtsh@163.com

*Contributed equally

Abbreviations: miRNAs, microRNAs; DDP, cisplatin

Key words: microRNA-20a, nuclear factor $\kappa \mathrm{B}$, cylindromatosis, cisplatin resistance, gastric cancer
CYLD, downregulated the expression levels of p65, livin and survivin, and led to a higher proportion of apoptotic cells in the SGC7901/DDP cells. By contrast, ectopic expression of miR-20a significantly repressed the expression of CYLD, upregulated the expression levels of p65, livin and survivin, and resulted in a decrease in the apoptosis induced by DDP in the SGC7901 cells. Taken together, the results of the present study suggested that miR-20a directly repressed the expression of CYLD, leading to activation of the NFKB pathway and the downstream targets, livin and survivin, which potentially induced GC chemoresistance. Altering miR-20a expression may be a potential therapeutic strategy for the treatment of chemoresistance in $\mathrm{GC}$ in the future.

\section{Introduction}

Gastric cancer (GC) is one of the most common malignancies worldwide (1). Currently, rates of early detection and diagnosis for $\mathrm{GC}$ are low, therefore, chemotherapy remains one of the major treatment methods for GC. However, the effectiveness of chemotherapy is often limited by the development of drug resistance (2). Cytological mechanisms, which have contributed to the chemoresistance include enhanced DNA repair activity, alterations in cell cycle and proliferation, and defective apoptosis (3). In addition, molecular mechanisms, including increased rates of drug efflux, alterations in drug metabolism and mutation of drug targets may be important in drug resistance $(4,5)$. Previous studies have found that the activation of certain signaling pathways, including the, phosphatase and tensin homolog/phosphoinositide 3-kinase/AKT, nuclear factor $(\mathrm{NF}) \kappa \mathrm{B} /$ inhibitor of $\mathrm{NF} \kappa \mathrm{B}, \mathrm{Ras} / \mathrm{Raf} / \mathrm{mitogen}$-activated protein kinase kinase and P53/murine double minute-2 (6-9), are also associated with drug resistance.

MicroRNAs (miRNAs), a class of short, non-coding RNAs of 19-22 nucleotides in length, function as post-transcriptional regulators by directly cleaving target messenger RNA or inducing translational repression (10). Previously, aberrant miRNAs have been found to be involved in drug resistance in different types of tumor, acting as oncogenes or tumor suppressors (11-14). Accumulating evidence has revealed that miRNA regulates NFkB signaling (15), and activation of the 
$\mathrm{NF} \kappa \mathrm{B}$ pathway enhances cell resistance to chemotherapeutic drugs (16). Therefore, the present study hypothesized that miRNA may induce chemoresistance through activation of the NFкB pathway.

In the present study, the potential function of miR-20a in GC chemoresistance was investigated. It was found that miR-20a was significantly upregulated in samples from patients with GC, particularly in those with chemoresistance. miR-20a was also found to be upregulated in a cisplatin (DPP)-resistant GC cell line (SGC7901/DDP.) miR-20a contributed to DDP resistance when overexpressed in the SGC7901 GC cell line. Furthermore, the present study demonstrated that miR-20a may activate the $\mathrm{NF} \kappa \mathrm{B}$ pathway and upregulate the downstream targets, survivin and livin, by repressing the expression of cylindromatosis (CYLD), leading to the resistance to DDP. The results of the study may aid the development of personalized treatment for patients with GC that exhibit abnormal levels of miR-20a.

\section{Materials and methods}

Clinical sample collection. A total of 121 patients (age range, 46-75; males, 66; females, 45) with histopathologically confirmed GC and 56 healthy donors (age range, 35-71; males, 33; females, 23) were recruited between 2012 and 2014 at the First Affiliated Hospital of Nanjing Medical University (Nanjing, China). A total of 28 GC plasma samples and an additional 28 formalin-fixed paraffin embedded (FFPE) sections of GC were obtained from preoperative patients who had not received chemotherapy. In addition, $30 \mathrm{GC}$ plasma samples and an additional 35 FFPE sections of GC were collected from patients with advanced disease, who had undergone two cycles of platinum-based chemotherapy. Response to chemotherapy was graded by the standards of Evaluation Criteria in Solid Tumors recommended by the World Health Organization (WHO), defined as complete remission (CR), partial remission (PR), stable disease (SD), and progressive disease (PD). All procedures were approved by the Institutional Review Boards of the First Affiliated Hospital of Nanjing Medical University. Written informed consent was obtained from each participant.

Cell culture and transfection. The SGC7901 human gastric cancer cell line was purchased from the National Institute of Cells (Shanghai, China). The DPP-resistant variant (SGC7901/DDP) was obtained from KeyGen Biotechnology Company (Nanjing, China). All cells were maintained in RPMI-1640 medium supplemented with $10 \%$ fetal calf serum (Gibco; Thermo Fisher Scientific, Inc., Waltham, MA, USA) in a humidified atmosphere with $5 \% \mathrm{CO}_{2}$ at $37^{\circ} \mathrm{C}$, as previously described $(17,18)$. To maintain the DPP-resistant phenotype, DDP, with final concentration of $1 \mu \mathrm{g} / \mathrm{ml}$, was added to the culture media of the SGC7901/DDP cells.

miRNA mimics and their appropriate negative control were obtained from Guangzhou RiboBio Co., Ltd. (Guangzhou, China). All transfection experiments were performed using Lipofectamine 2000 reagent (Invitrogen; Thermo Fisher Scientific, Inc.), according to the manufacturer's protocol. UCSC Genome Browser (genome.ucsc.edu/) was used to search the promoter regions of miR-20a.
Bioinformatics analysis. TargetScan (www.targetscan.org), a bioinformatics tool for miRNA target screening, was used to predict the target genes of miR-20a.

RNA isolation and reverse transcription-quantitative polymerase chain reaction $(R T-q P C R)$ analysis. Following tissue homogenization, total RNA from the cells was extracted using TRIzol reagent (Invitrogen; Thermo Fisher Scientific, Inc.), according to the manufacturer's protocol. For the plasma samples, total RNA was extracted using the mirVana Paris kit (Ambion, Austin, TX, USA), according to the manufacturer's protocol. Total RNA from the FFPE tissues was isolated using the High Pure FFPE RNA Micro kit (Ambion), according to the manufacturer's protocol. The amplification of miRNA was performed using the specific primers for the RT-qPCR analysis in the Bulge-Loop ${ }^{\mathrm{TM}}$ miRNA qRT-PCR primer set (RiboBio Co., Ltd.), as previously described $(19,20)$. RT-qPCR was performed, according to the manufacturer's protocol. The expression levels of the PCR products were determined by the level of fluorescence emitted by SYBR Green $\left(S\right.$ YBR ${ }^{\circledR}$ Premix Ex Taq ${ }^{\mathrm{TM}}$ II, Takara Bio, Inc., Otsu, Japan). The relative expression levels of the target miRNAs from the cells and tissue samples were calculated using the comparative $2^{-\Delta \Delta \mathrm{Cq}}$ method, relative to U6 snRNA, as previously described $(17,18)$. The levels of miRNA in the plasma samples were determined based on a standard curve constructed with using synthetic miRNAs (micrON miRNA mimic; RiboBio Co., Ltd.), as previously described $(19,20)$.

In vitro drug sensitivity assay. The SGC7901 cells and SGC7901/DDP cells were seeded into 6-well plates $\left(6 \times 10^{5}\right.$ cells/well). Either the miR-20a mimic $(100 \mathrm{nM})$ or mimic control $(100 \mathrm{nM})$ were transfected into the SGC7901 cells, whereas the SGC7901/DDP cells were transfected with either miR-20a inhibitor $(100 \mathrm{nM})$ or inhibitor control (100 nM). The miR-20a mimic, inhibitor and relative controls were purchased from Gene Pharma (Shanghai, China).

At $24 \mathrm{~h}$ post-transfection, the cells were seeded into 96-well plates $\left(5 \times 10^{3}\right.$ cells/well). Following cellular adhesion, freshly prepared DDP was added at final concentrations 10, 1, 0.1 and 0.01 times that of the human peak plasma concentration for DDP $(2.0 \mu \mathrm{g} / \mathrm{ml})$. After $48 \mathrm{~h}, 3$-(4,5-dimethylthiazol-2-yl)-2,5-diphenyl-tetrazolium bromide (MTT) was added to each well to measure the viability of the cells, and the plate was incubated for $2 \mathrm{~h}$ in a humidified incubator. The absorbance at $490 \mathrm{~nm}$ of each well was measured on a spectrophotometer. The relative survival curve was used to calculate the concentration of cisplatin, which caused $50 \%$ inhibition of growth.

Dual-luciferase activity assay. The 3'-untranslated region (3'UTR) sequences of human CYLD cDNA containing the predicted target site of miR-20a, were chemically synthesized by RiboBio Co., Ltd. HEK293T human embryonic kidney cells (Cell Bank of the Chinese Academy of Sciences, Shanghai, China) were plated in 24-well plates $\left(1.5 \times 10^{5}\right.$ cells/well) for $24 \mathrm{~h}$, following which $200 \mathrm{ng}$ of the pGL3-CYLD-3'-UTR, 60 pmol of the miR-20a mimic or miRNA mimic control, and $80 \mathrm{ng}$ of pRL-TK (Promega Corporation, Madison, WI, USA) were co-transfected into 


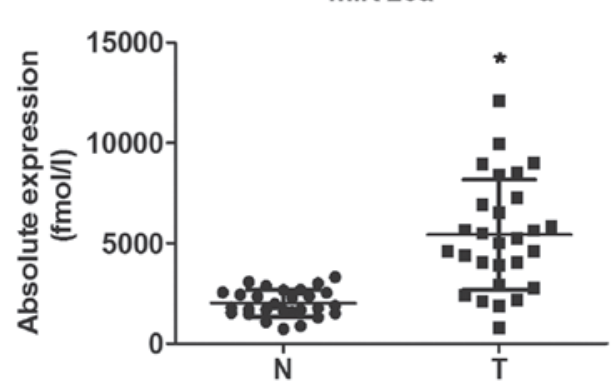

B

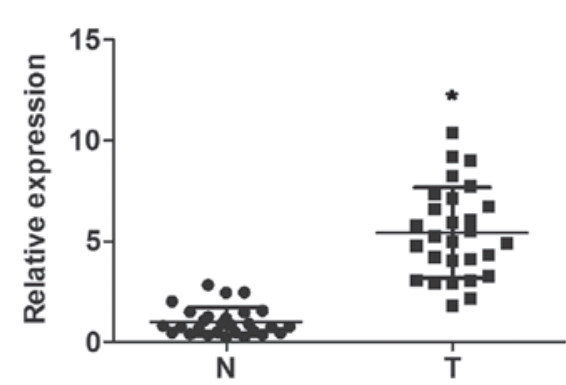

Figure 1. miR-20a is significantly upregulated in GC plasma and GC tissue samples. (A) Expression levels of miR-20a were detected in 28 normal plasma samples and 28 preoperative GC plasma samples using reverse transcription-quantitative polymerase chain reaction analysis. Expression levels of miR-20a in the tumor group were higher, compared with those in the normal group. Y-axis data are presented as log10 (concentration; fmol/1). (B) Expression levels of miR-20a were also upregulated in GC formalin-fixed paraffin embedded samples. Y-axis data are presented as relative expression (normalized to U6; calculated using the $2^{-\triangle \Delta C q}$ method). Data are expressed as the mean \pm standard deviation. * $\mathrm{P}<0.01$. N, normal; T, tumor; GC, gastic cancer; miR, microRNA.

the cells. Luciferase activity was measured using the Dual Luciferase Reporter Assay system (Promega Corporation) consecutively. The firefly luciferase activity was normalized to the expression of renilla luciferase.

Immunohistochemistry. For immunohistochemical analysis, tissue sections (4- $\mu$ m-thick) were formalin-fixed and paraffin-embedded, and were stained using the avidin-biotin complex method (21). Antigen retrieval was performed using microwave irradiation in $10 \mathrm{mM}$ citrate buffer $(\mathrm{pH} 6.0$ ), followed by incubation with primary antibodies at $4^{\circ} \mathrm{C}$ overnight. The antibody for CYLD (cat. no. 8462; dilution 1:200) was purchased from Cell Signaling Technology, Inc. (Danvers, MA, USA). The biotinylated goat anti-rabbit secondary antibody (cat. no. A0227 ) was purchased from Beyotime Institute of Biotechnology (Shanghai, China). Specimins were incubated in the secondary antibody (1:50) for $30 \mathrm{~min}$ at room temperature. Staining was repeated if the result was uncertain. The slides were scored independently by two observers blinded to the clinicopathological characteristics. The immunostaining of the slides was evaluated under an optical microscope (magnification x400). Discordant scores were reevaluated to reach a consensus. The staining intensity of the expression of CYLD was scored (scores 0-3 for negative, weak, moderate and strong expression, respectively), as was the percentage of positive cancer cells ( 0 for $0 \%$; 0.1 for $1-9 \%$; 0.5 for $10-49 \%$; and 1.0 for $\geq 50 \%$ ). The staining intensity was multiplied by the proportion score of the percentage of positive cancer cells.

Western blot analysis. Total protein was prepared from the cells using radioimmunoprecipitation assay buffer in the presence of proteinase inhibitor. The nuclear proteins were extracted from the cells using a nuclear cytoplasmic extraction kit (Beyotime Institute of Biotechnology). Protein levels were quantified using bicinchoninic acid assay (Beyotime Institute of Biotechnology). Western blot analysis was performed, as previously described $(17,18)$. The primary antibodies for CYLD (cat. no. 8462), P65 (cat. no. 8242), livin (cat. no. 5471), H3 (cat. no. 4499) and survivin (cat. no. 2808) were obtained from Cell Signaling Technology, Inc. The blots were incubated with the indicated primary antibodies or GAPDH antibody (cat. no. AP0063; Bioworld Technology, Inc., St. Louis Park,
$\mathrm{MN}, \mathrm{USA}$ ) overnight at $4^{\circ} \mathrm{C}$, then with goat anti-rabbit horseradish peroxidase (HRP)-conjugated antibody (cat. no. sc-2030; Santa Cruz Biotechnology, Inc., Dallas, TX, USA) at room temperature for $1 \mathrm{~h}$. The protein bands were visualized using an Immobilon Western Chemiluminescent HRP Substrate kit (Beyotime Institute of Biotechnology,) and a ChemiDoc XRS+ Imaging system (Bio-Rad Laboratories, Inc., Hercules, CA, USA). Grey value analysis of protein bands was performed for quantification of the protein levels using Image $\mathbf{J}$ software (version 1; National Institutes of Health, Bethesda, MD, USA). The levels of total protein were normalized to total GAPDH, and nuclear protein to H3. The fold changes were calculated.

Apoptosis assay. The cells were transfected with miR-20a mimic or inhibitor, respectively. After $24 \mathrm{~h}$, the cells were treated with DDP at a final concentration of $10 \mu \mathrm{g} / \mathrm{ml}$. After $48 \mathrm{~h}$, apoptosis was assessed via the counting of annexin $\mathrm{V}$-fluorescein isothiocyanate-positive and propidium iodide-negative cells using flow cytometry, as described previously $(17,18)$.

Statistical analysis. Data are presented as the mean \pm standard deviation from at least three separate experiments. Two-tailed Student's $t$-tests were performed for comparisons using SPSS (version 15.0; SPSS, Inc., Chicago, IL, USA) software. Spearman's non-parametric correlation test was performed to analyze the correlation between the expression levels of miR-186 and Twist1 by GraphPad Prism 5 (GraphPad Software, Inc., La Jolla, CA, USA). P $<0.05$ was considered to indicate a statistically significant difference.

\section{Results}

miR-20a is significantly upregulated in GC plasma samples and GC tissue samples. To determine the basal expression of miR-20a in GC, the present study first performed RT-qPCR analysis to examine the plasma levels of miR-20a in 28 patients with preoperative GC and 28 healthy subjects. The expression of miR-20a was significantly increased in the GC group, compared with the normal group (Fig. 1A). Furthermore, it was found that the expression levels of miR-20a were significantly higher in the 28 GC FFPE tissue samples, compared with those in the 28 samples of normal gastric tissue (Fig. 1B). 
miR-20a is involved in GC chemotherapeutic resistance. To further investigate the potential role of miR-20a in GC chemoresistance, 30 plasma samples and 35 FFPE sections were collected from patients with advanced GC who had undergone two cycles of platinum-based chemotherapy. The response to chemotherapy was evaluated using standard WHO criteria, of CR, PR, SD and PD. According to these criteria, the plasma samples showed that 10 patients had PR, nine patients had SD and 11 patients had PD, with no patients in CR. In the tissue specimens, 12 had PR, 11 had SD and 12 had PD, with no cases of CR. The expression of plasma miR-20a in the PD+SD group were significantly higher, compared with that in the PR group (Fig. 2A). The upregulation of miR-20a was also detected in the FFPE tissues of the PD+SD group, compared with the PR group (Fig. 2B). The results of the RT-qPCR analysis revealed that the expression of miR-20a was also significantly increased in the SGC7901/DDP cells, compared with the parental SGC7901 cell line (Fig. 2C), which was consistent with the results obtained in the clinical GC samples. These results revealed that the overexpression of miR-20a was significantly associated with GC chemoresistance.

miR-20a regulates resistance to DDP in the SGC7901/DDP cell line. To examine the functional role of miR-20a in the chemoresistance of GC, the miR-20a mimic or inhibitor were transfected into the SGC7901 cells or SGC7901/DDP cells respectively. Prior to the miRNA transfection, the significant resistance of the SGC7901/DDP cells to DDP, compared with the parental SGC7901 cells was verified. The MTT assay revealed reduced sensitivity of the SGC7901 cells transfected with the miR-20a mimic to DDP, compared with that of the cells transfected with the mimic control (Fig. 3A). By contrast, transfection with the miR-20a inhibitor markedly enhanced the sensitivity of the SGC7901/DDP cells to DDP, compared with the control (Fig. 3B). Taken together, these results indicated that miR-20a modulated the resistance of the SGC7901/DDP cells to DDP.

CYLD is a direct target of miR-20a. Using TargetScan software for miRNA target prediction, the present study found CYLD as a potential direct target gene of miR-20a. To assess the relevance of miR-20a/CYLD, immunohistochemistry was performed to examine the expression of CYLD in $28 \mathrm{GC}$ tissue samples. The expression of miR-20a was also analyzed uisng RT-qPCR analysis in the same specimens. Among these 28 specimens, using the median expression value of miR-20a as a cutoff point, the cohort was divided into miR-20a-high or miR-20a-low tumors. The protein expression levels of CYLD in the miR-20a-high group were significantly lower, compared with those in the miR-20a-low group (Fig. 4A). An inverse correlation $\left(\mathrm{R}^{2}=0.4574 ; \mathrm{P}<0.001\right)$ between the expression of miR-20a and the protein levels of CYLD was observed using Spearman's correlation analysis (Fig. 4B). In addition, western blot analysis revealed that CYLD was significantly decreased at the protein level in the SGC7901/DDP cells, compared with the parental SGC7901 cell line (Fig. 4C).

To further confirm whether CYLD was a target for miR-20a, luciferase reporter plasmids containing the 3'UTR of CYLD were constructed and co-transfected with miR-20a mimic in the HEK293T human embryonic kidney cell line. The results showed that luciferase activity was significantly decreased in

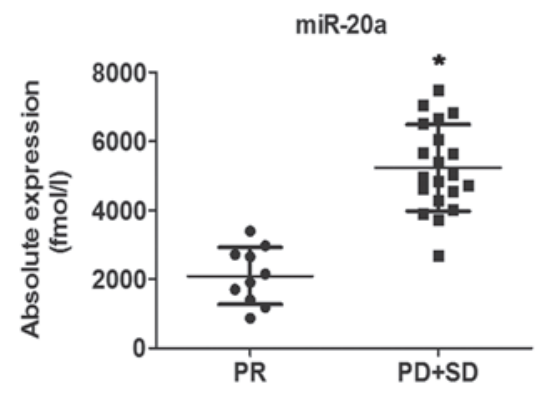

B

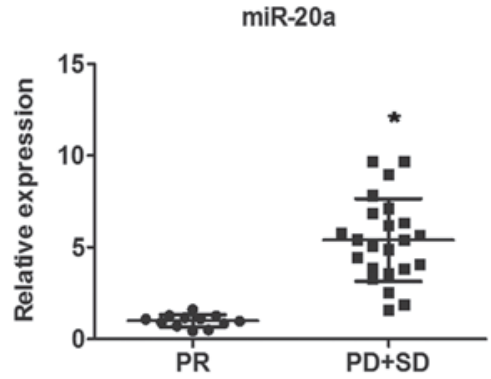

C

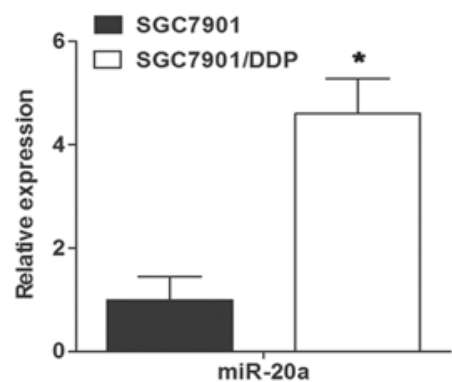

Figure 2. miR-20a is involved in gastric cancer chemotherapeutic resistance. (A) Plasma levels of miR-20a were significantly higher in the PD+SD samples, compared with the PR samples. (B) Expression levels of miR-20a in the formalin-fixed paraffin embedded tissues were upregulated in the PD+SD group, compared with the PR group. (C) Expression levels of miR-20a were also significantly upregulated in SGC7901/DDP cells, compared with the parental SGC7901 cell line. Data are presented as the mean \pm standard deviation. "P<0.01. miR, microRNA; PD, progressive disease; $\mathrm{D}$, stable disease; $\mathrm{PR}$, partial remission; DDP, cisplatin.

the cells transfected with the miR-20a mimic, compared with the vector control (Fig. 4D). These results showed that miR-20a may negatively regulate the expression of CYLD by directly targeting its 3'UTR.

miR-20a represses the expression of $C Y L D$, leading to $N F \kappa B$ activation and upregulation of the downstream targets, survivin and livin. In the present study, the upregulation of miR-20a in the SGC7901/DDP cells was concurrent with the downregulation of CYLD, compared with the parental SGC7901 cells. In addition, the $\mathrm{NF} \kappa \mathrm{B}$ pathway-associated proteins, $\mathrm{p} 65$, livin and survivin, showed significantly higher levels of expression in the SGC7901/DDP cells, compared with the SGC7901 cells (Fig. 5A). The present study verified that CYLD was a direct 

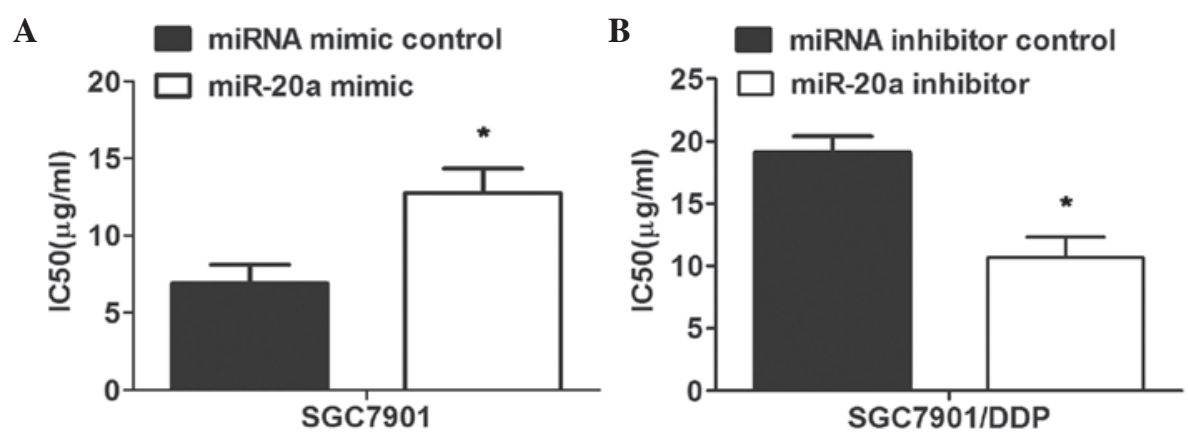

Figure 3. miR-20a regulates DDP resistance of the SGC7901/DDP cell line. (A) A 3-(4,5-dimethylthiazol-2-yl)-2,5-diphenyl-tetrazolium bromide assay revealed that the sensitivity of SGC7901 cells transfected with miR-20a mimic to DDP was decreased, compared with that in those transfected with the mimic control. (B) In SGC7901/DDP cells, the miR-20a inhibitor significantly increased the sensitivity to DDP. Data are presented as the mean \pm standard deviation. "P<0.01. DDP, cisplatin; miR; microRNA; IC50, 50\% inhibition of growth.

A

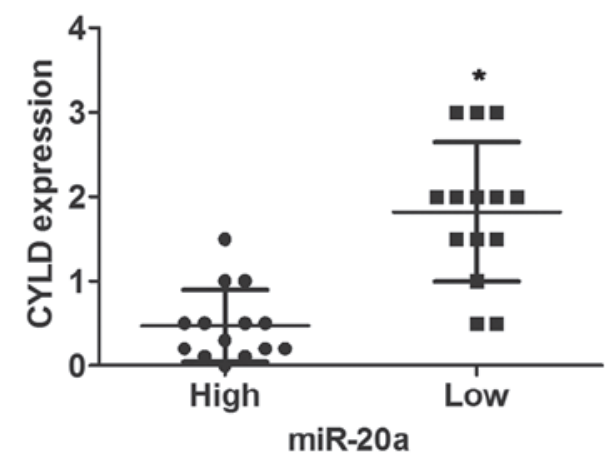

C

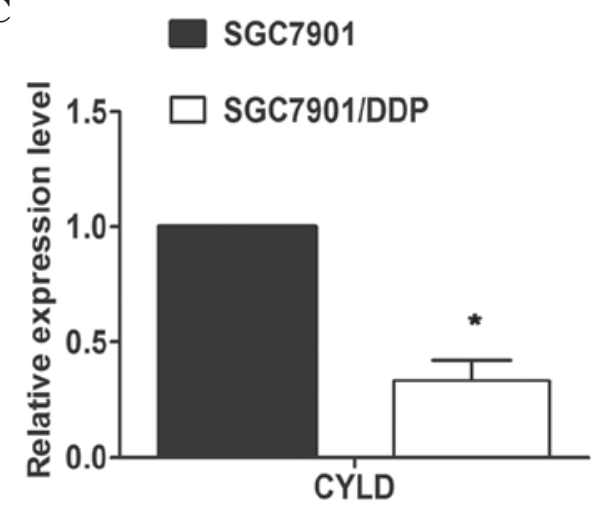

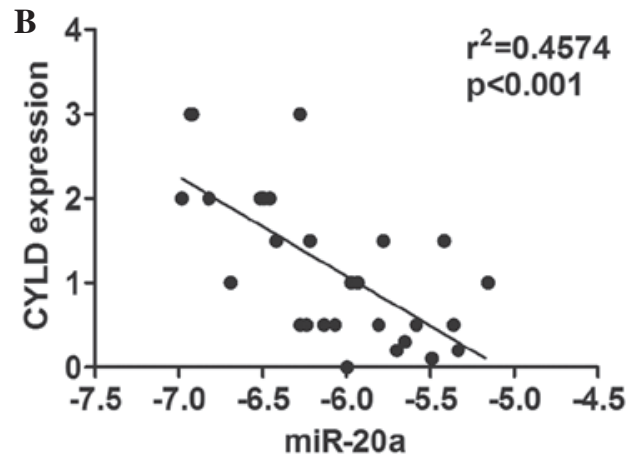

D

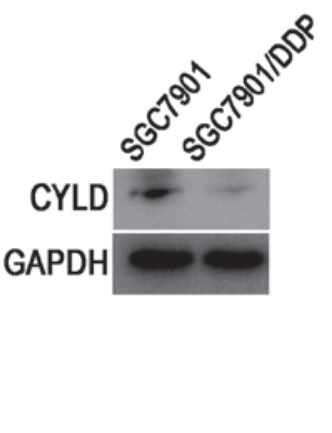

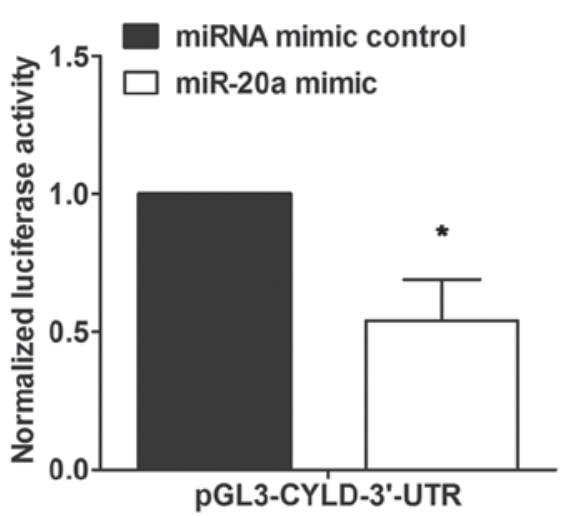

Figure 4. miR-20a directly regulates the expression of CYLD. (A) Expression of CYLD and miR-20a were inversely correlated in GC tissues (n=28). (B) A plot of the relative expression of miR-20a, vs. CYLD showed an inverse correlation between the two. The correlation index $\mathrm{R}^{2}$ was calculated using Spearman's rank test $\left(\mathrm{R}^{2}=0.4574 ; \mathrm{P}<0.001\right)$. Y-axis data are presented as immunohistochemistry scores, $\mathrm{X}$-axis data are presented as relative expression (normalized to U6; calculated using the $2^{-\Delta \Delta C q}$ method). (C) Protein expression levels of CYLD in the SGC7901/DDP cells and the parental SGC7901 cell line. (D) Luciferase assay showed that co-transfection of CYLD 3'UTR and miR-20a decreased the relative luciferase activity in 293T cells. Data are presented as the mean \pm standard deviation. "P<0.01. miR, microRNA; CYLD, cylindromatosis; 3'-UTR, 3'-untranslated region; DDP, cisplatin.

target of miR-20a, and also a negative regulator of the NFkB pathway. Thus, it was hypothesized that miR-20a may activate the NFKB pathway by repressing the expression of CYLD. To confirm this, the SGC7901/DDP cells and SGC7901 cells were transfected with miR-20a inhibitor or mimic, respectively, and western blot analysis was performed to examine the expression of these proteins. In the SGC7901/DDP cells, at $72 \mathrm{~h}$ post-transfection, the results demonstrated that the reduced level of miR-20a increased the expression of CYLD, but decreased the expression of p65, survivin and livin (Fig. 5B). By contrast, the expression of CYLD in the SGC7901 cells transfected with the miR-20a mimic was downregulated, whereas the expression levels of p65, survivin and livin were upregulated (Fig. 5C). These results suggested that miR-20a repressed the expression of CYLD, leading to activation of the NFKB pathway and upregulation of the downstream targets, livin and survivin.

Inhibition of miR-20a sensitizes SGC7901/DDP cells to DDP-induced apoptosis. The overexpression of anti-apoptotic proteins, including livin and survivin, has been reported to contribute to resistance to drug-induced apoptosis in several types of cancer $(17,18,22)$. In the present study, it was 
A
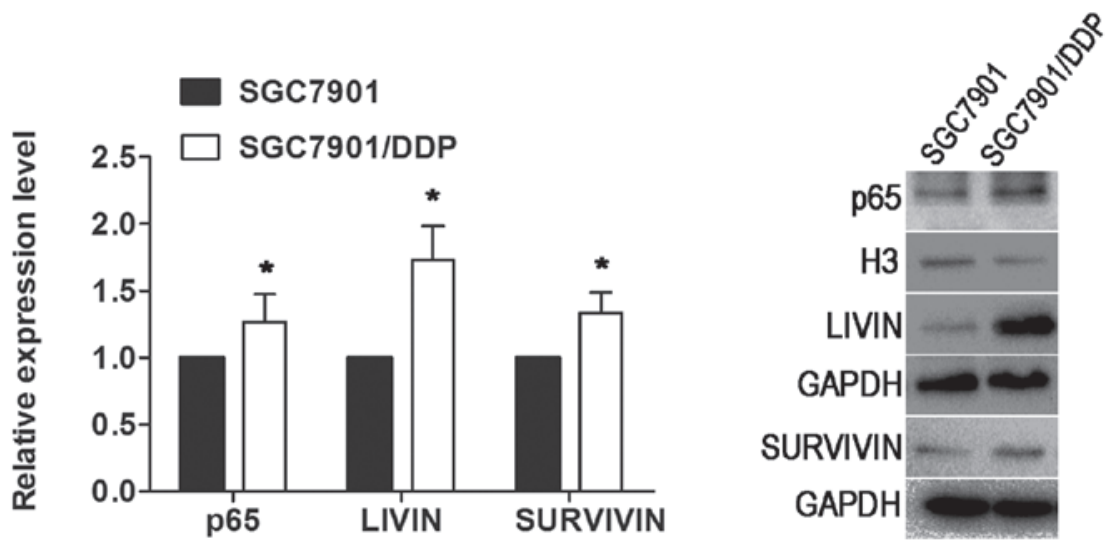

B

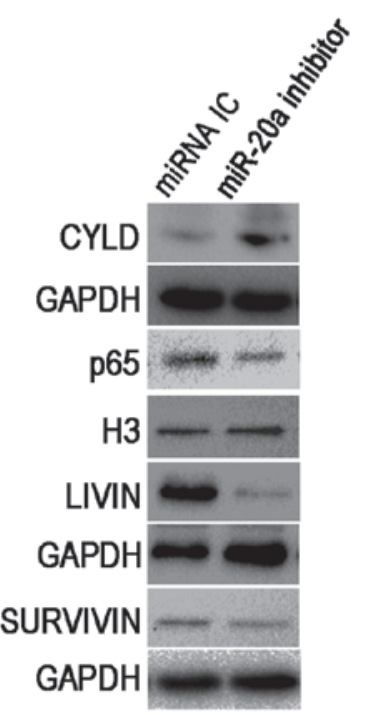

C
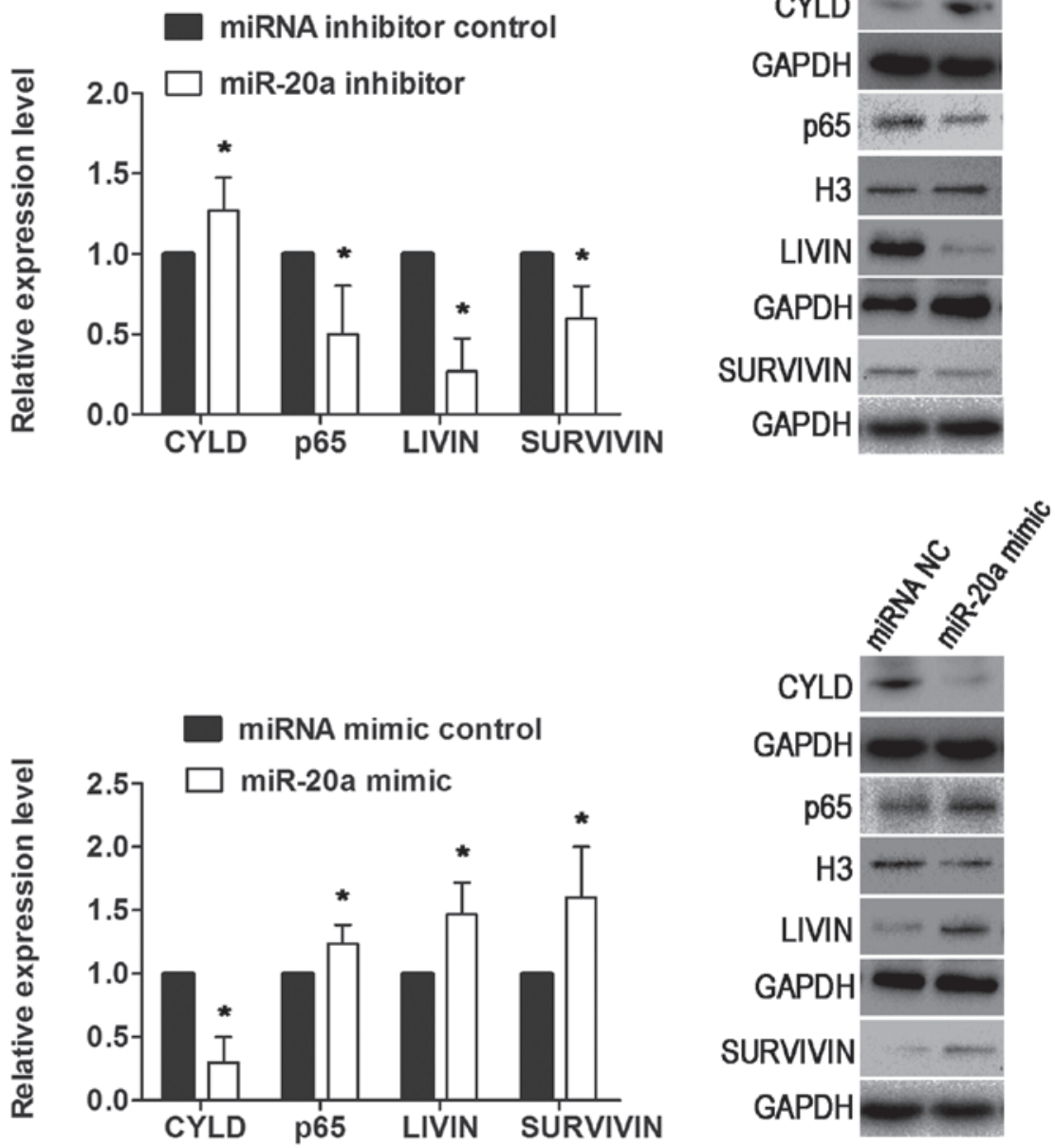

Figure 5. miR-20a represses the expression of CYLD, leading to NF $\kappa B$ activation and the upregulation of the downstream targets, survivin and livin (A) Western blot analysis showed that p65, livin and survivin were upregulated in the SGC7901/DDP cells. The levels of p65 in the nuclei were normalized to H3. (B) In SGC7901/DDP cells transfected with miR-20a inhibitor, at $72 \mathrm{~h}$ post-transfection, the results revealed that low levels of miR-20a increased the expression of CYLD, and decreased the expression levels of p65, livin and survivin. (C) Expression of CYLD in the SGC7901 cells transfected with miR-20a mimic was downregulated, whereas the expression levels of $\mathrm{p} 65$, livin and survivin were upregulated. Data are presented as the mean \pm standard deviation. "P<0.01. NC, mimic control; IC, inhibitor control; miRNA/miR, microRNA; CYLD, cylindromatosis; DDP, cisplatin.

demonstrated that the overexpression of miR-20a may enhance the resistance of GC cells to DDP, at least in part, via the activation of $\mathrm{NFKB}$ and its downstream targets, livin and survivin. Thus, it was hypothesized that miR-20a may also be associated with DDP resistance by regulating the apoptosis of GC cells. To further confirm this, the SGC7901/DDP and SGC7901 cells were transfected with the inhibitor or mimic of miR-20a, respectively, followed by the analysis of DDP-induced apoptosis. The 
A

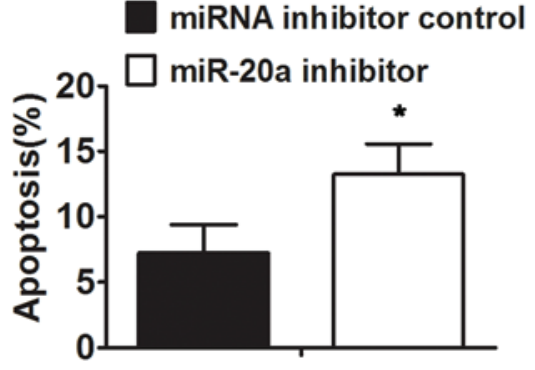

miRNA inhibitor control

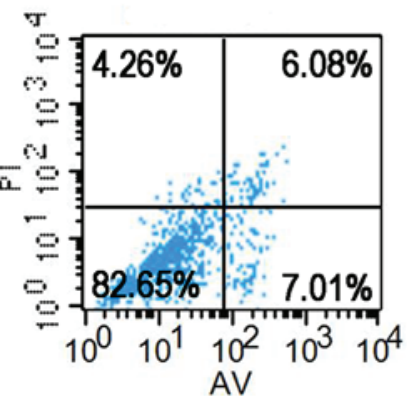

miRNA mimic control

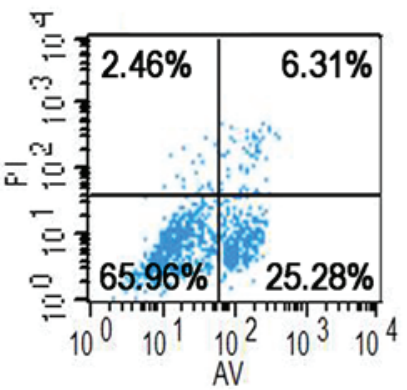

miR-20a inhibitor
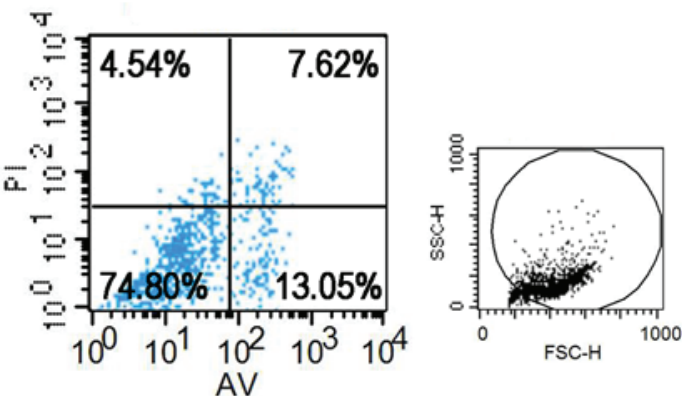

miR-20a mimic

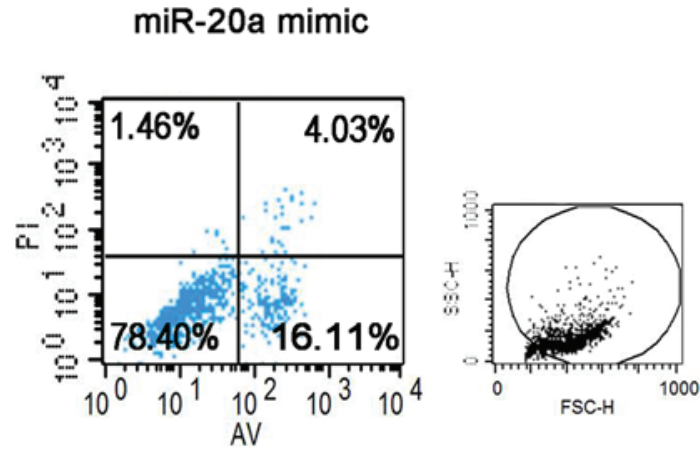

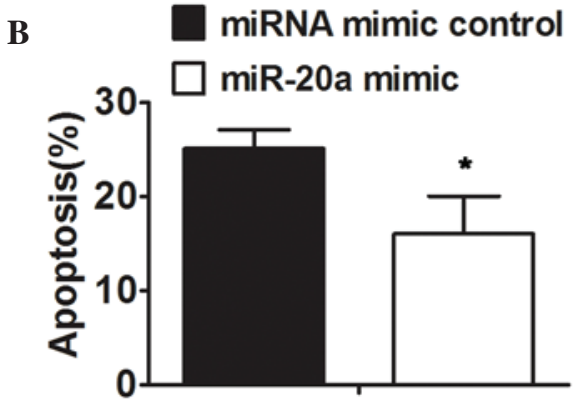

Figure 6. Inhibition of miR-20a sensitizes SGC7901/DDP cells to DDP-induced apoptosis. (A) SGC7901/DDP cells were transfected with miR-20a inhibitor or control following DDP treatment. An increased number of cells undergoing apoptosis were found in the cells with downregulated expression of miR-20a. (B) SGC7901 cells were transfected with the miR-20a mimic or control. There were fewer cells undergoing apoptosis in the cells overexpressing miR-20a. Representative flow cytometry results are shown adjacent to the graphs. Data are presented as the mean \pm standard deviation. ${ }^{*} \mathrm{P}<0.01$. miR/miRNA, microRNA; DDP, cisplatin; PI, propidium iodide; AV, avidin.

results indicated that there was a higher proportion of apoptotic cells in the SGC7901/DDP cells transfected with miR-20a inhibitor, compared with those transfected with the inhibitor control following DDP treatment (Fig. 6A). By contrast, the ectopic expression of miR-20a resulted in a decrease in the apoptosis induced by DDP in the SGC7901 cells (Fig. 6B).

\section{Discussion}

miR-20a is a member of the miR-17-92 cluster and acts as an oncogene. It has been demonstrated that miR-20a is upregulated, and promotes tumorigenesis and cancer progression in diverse cancer subtypes, including cervical cancer (23), ovarian cancer (24), osteosarcoma (25), anaplastic thyroid cancer (26) and nasopharyngeal carcinoma (27). A study by Li et al (28) revealed that miR-20a was markedly increased in GC tissues and cell lines, and that the ectopic expression of miR-20a promoted the proliferation, migration and invasion of GC cells. A study by Chai et al (29) suggested that miR-20a targeted BNIP2 and contributed to chemotherapeutic resistance in colorectal adenocarcinoma cell lines. In the present study, it was confirmed that miR-20a was significantly upregulated in SGC7901/DDP cells and was associated with GC chemoresistance. In addition, miR-20a regulated the resistance of the SGC7901/DDP cell line to DDP.

Several studies have shown that constitutively activated NFKB signaling and upregulation of drug resistance-associated proteins, including B cell lymphoma-2, myeloid cell leukemia-1, multidrug resistance 1 and X-linked inhibitor of apoptosis protein, are critical in mediating resistance in various types of cancer $(9,30,31)$. CYLD, a deubiquitination enzyme, acts as a tumor suppressor in different types of cancer. Currently, CYLD is known to function as a negative regulator of the NFאB signaling pathway, and is closely involved in regulating the apoptosis of cancer cells. The ubiquitination of key signaling molecules by E3 ubiquitin ligases is required in $\mathrm{NF} \kappa \mathrm{B}$ signaling transmission, whereas $\mathrm{NF \kappa B}$ activation is limited by ubiquitin deconjugation induced by deubiquitinases, including CYLD (32-35). In addition, previous investigations have suggested that inhibition of the $\mathrm{NF \kappa B}$ pathway sensitized cells to chemotherapeutic drugs in GC $(36,37)$. In the present study, it was found that miR-20a directly targeted CYLD, demonstrating a novel mechanism of CYLD dysregulation in GC. Further investigation was performed to determine whether there was a correlation between miR-20a and the NFאB pathway, to clarify the effects of miR-20a on $\mathrm{GC}$ chemoresistance. It was found that the overexpression of miR-20a in the SGC7901/DDP cells was concurrent with the downregulation of CYLD and the upregulation of $\mathrm{p} 65$. The expression level of CYLD was upregulated and the expression of p65 was downregulated when the SGC7901/DDP cells were transfected with the miR-20a inhibitor. In the SGC7901 cells, the ectopic expression of miR-20a decreased the expression of CYLD and increased the expression of p65. The aforementioned results indicated that miR-20a was essential, at least in part, for activating the $\mathrm{NF} \kappa \mathrm{B}$ pathway in GC. Acting as an upstream regulator of the $\mathrm{NF \kappa B}$ pathway, the downregulation of miR-20a may be vital in NFkB pathway suppression. 
Livin and survivin, members of the inhibitor of apoptosis protein family, function as anti-apoptotic factors and are important in inhibiting apoptosis (38). The overexpression of inhibitor of apoptosis is correlated with cancer chemoresistance. The present study suggested that miR-20a regulated the expression levels of livin and survivin via NFkB signaling. The inhibition of miR-20a rendered the SGC7901/DDP cells more sensitive to DDP-induced apoptosis. It was hypothesized that miR-20a may also be involved in the development of DDP resistance by modulating the apoptosis of GC cells.

However, the mechanism of miR-20a upregulation was not exact. Using the UCSC database, the present study found that $\mathrm{NF} \mathrm{KB}$ binding sites were present in the promoter regions of miR-20a, which indicated that $\mathrm{NF} \kappa \mathrm{B}$ may directly regulate the expression of miR-20a. A study by Zhou et al (39) supported this. Therefore, it was hypothesized that the positive feedback loop, miR-20a/CYLD/NFkB/miR-20a, was an important mechanism for the sustained activation of $\mathrm{NF \kappa B}$ in drug-resistant GC cells. However, further investigation is required to confirm this.

In conclusion, the present study provided the first evidence, to the best of our knowledge, that miR-20a directly targeted CYLD, resulting in activation of the NFKB pathway and the downstream targets, livin and survivin, which potentially contributed to GC chemoresistance. These results may provide theoretical information for miR-20a as a key target to reverse chemotherapy resistance. However, the results of the present study were obtained from cell lines and do not necessarily reflect the actual surrogates for clinical tumors. Therefore, further investigation is required to assess the role of miR-20a in vivo and clinically.

\section{Acknowledgements}

The authors are grateful for the funding support by the National Natural Science Foundation of China (grant nos. 81201705 and 81201796) and the Natural Science Foundation of Jiangsu Province (grant no. BK2012442).

\section{References}

1. Ferlay J, Soerjomataram I, Dikshit R, Eser S, Mathers C, Rebelo M, Parkin DM, Forman D and Bray F: Cancer incidence and mortality worldwide: Sources, methods and major patterns in GLOBOCAN 2012. Int J Cancer 136: E359-E386, 2015.

2. Orditura M, Galizia G, Sforza V, Gambardella V, Fabozzi A, Laterza MM, Andreozzi F, Ventriglia J, Savastano B, Mabilia A, et al: Treatment of gastric cancer. World J Gastroenterol 20: 1635-1649, 2014.

3. Lippert TH, Ruoff HJ and Volm M: Intrinsic and acquired drug resistance in malignant tumors. The main reason for therapeutic failure. Arzneimittelforschung 58: 261-264, 2008.

4. Holohan C, Van Schaeybroeck S, Longley DB and Johnston PG: Cancer drug resistance: An evolving paradigm. Nat Rev Cancer 13: 714-726, 2013

5. Longley DB and Johnston PG: Molecular mechanisms of drug resistance. J Pathol 205: 275-292, 2005.

6. Nakagawa Y, Sedukhina AS, Okamoto N, Nagasawa S, Suzuki N, Ohta T, Hattori H, Roche-Molina M, Narváez AJ, Jeyasekharan AD, et al: NF- $\mathrm{BB}$ signaling mediates acquired resistance after PARP inhibition. Oncotarget 6: 3825-3839, 2015.

7. Halilovic E, She QB, Ye Q, Pagliarini R, Sellers WR, Solit DB and Rosen N: PIK3CA mutation uncouples tumor growth and cyclin D1 regulation from MEK/ERK and mutant KRAS signaling. Cancer Res 70: 6804-6814, 2010.
8. Michaelis M, Rothweiler F, Barth S, Cinatl J, van Rikxoort M, Löschmann N, Voges Y, Breitling R, von Deimling A, Rödel F, et al: Adaptation of cancer cells from different entities to the MDM2 inhibitor nutlin-3 results in the emergence of p53-mutated multi-drug-resistant cancer cells. Cell Death Dis 2: e243, 2011.

9. Lin $X$, Zhang $X$, Wang Q, Li J, Zhang $P$, Zhao $M$ and Li X: Perifosine downregulates MDR1 gene expression and reverses multidrug-resistant phenotype by inhibiting $\mathrm{PI} 3 \mathrm{~K} / \mathrm{Akt} / \mathrm{NF}-\kappa \mathrm{B}$ signaling pathway in a human breast cancer cell line. Neoplasma 59: 248-256, 2012.

10. Bartel DP: MicroRNAs: Genomics, biogenesis, mechanism and function. Cell 116: 281-297, 2004.

11. Xiang Y, Ma N, Wang D, Zhang Y, Zhou J, Wu G, Zhao R, Huang H, Wang X, Qiao Y, et al: MiR-152 and miR-185 co-contribute to ovarian cancer cells cisplatin sensitivity by targeting DNMT1 directly: A novel epigenetic therapy independent of decitabine. Oncogene 33: 378-386, 2014.

12. Shang Y, Zhang Z, Liu Z, Feng B, Ren G, Li K, Zhou L, Sun Y, $\mathrm{Li} \mathrm{M}$, Zhou J, et al: MiR-508-5p regulates multidrug resistance of gastric cancer by targeting ABCB1 and ZNRD1. Oncogene 33: 3267-3276, 2014.

13. Sui C, Meng F, Li Y and Jiang Y: MiR-148b reverses cisplatin-resistance in non-small cell cancer cells via negatively regulating DNA (cytosine-5)-methyltransferase 1 (DNMT1) expression. J Transl Med 13: 132, 2015.

14. Fang L, Li H, Wang L, Hu J, Jin T, Wang J and Yang BB: MicroRNA-17-5p promotes chemotherapeutic drug resistance and tumour metastasis of colorectal cancer by repressing PTEN expression. Oncotarget 5: 2974-2987, 2014.

15. van Jaarsveld MT, Helleman J, Boersma AW, van Kuijk PF, van Ijcken WF, Despierre E, Vergote I, Mathijssen RH, Berns EM, Verweij J, et al: MiR-141 regulates KEAP1 and modulates cisplatin sensitivity in ovarian cancer cells. Oncogene 32: 4284-4293, 2013.

16. Eberle KE, Sansing HA, Szaniszlo P, Resto VA and Berrier AL: Carcinoma matrix controls resistance to cisplatin through talin regulation of NF-kB. PLoS One 6: e21496, 2011.

17. Zhu W, Xu H, Zhu D, Zhi H, Wang T, Wang J, Jiang B, Shu Y and Liu P: MiR-200bc/429 cluster modulates multidrug resistance of human cancer cell lines by targeting BCL2 and XIAP. Cancer Chemother Pharmacol 69: 723-731, 2012.

18. Zhu W, Shan X, Wang T, Shu Y and Liu P: MiR-181b modulates multidrug resistance by targeting BCL2 in human cancer cell lines. Int J Cancer 127: 2520-2529, 2010.

19. Zhao DS, Chen Y, Jiang H, Lu JP, Zhang G, Geng J, Zhang Q, Shen JH, Zhou X, Zhu W and Shan QJ: Serum miR-210 and miR-30a expressions tend to revert to fetal levels in Chinese adult patients with chronic heart failure. Cardiovasc Pathol 22: 444-450, 2013

20. Zhou X, Zhu W, Li H, Wen W, Cheng W, Wang F, Wu Y, Qi L, Fan Y, Chen Y, et al: Diagnostic value of a plasma microRNA signature in gastric cancer: A microRNA expression analysis. Sci Rep 5: 11251, 2015.

21. Bratthauer GL: The avidin-biotin complex (ABC) method and other avidin-biotin binding methods. Methods Mol Biol 588: 257-270, 2010.

22. Zhu DX, Zhu W, Fang C, Fan L, Zou ZJ, Wang YH, Liu P, Hong M, Miao KR, Liu P, et al: MiR-181a/b significantly enhances drug sensitivity in chronic lymphocytic leukemia cells via targeting multiple anti-apoptosis genes. Carcinogenesis 33: 1294-1301, 2012

23. Zhao S, Yao D, Chen J, Ding N and Ren F: MiR-20a promotes cervical cancer proliferation and metastasis in vitro and in vivo. PLoS One 10: e0120905, 2015.

24. Fan X, Liu Y, Jiang J, Ma Z, Wu H, Liu T, Liu M, Li X and Tang $\mathrm{H}$ : MiR-20a promotes proliferation and invasion by targeting APP in human ovarian cancer cells. Acta Biochim Biophys Sin (Shanghai) 42: 318-324, 2010.

25. Huang G, Nishimoto K, Zhou Z, Hughes D and Kleinerman ES: MiR-20a encoded by the miR-17-92 cluster increases the metastatic potential of osteosarcoma cells by regulating Fas expression. Cancer Res 72: 908-916, 2012.

26. Xiong Y, Zhang L and Kebebew E: MiR-20a is upregulated in anaplastic thyroid cancer and targets LIMK1. PLoS One 9: e96103, 2014

27. Zeng X, Xiang J, Wu M, Xiong W, Tang H, Deng M, Li X, Liao Q, Su B, Luo Z, et al: Circulating miR-17, miR-20a, miR-29c and miR-223 combined as non-invasive biomarkers in nasopharyngeal carcinoma. PLoS One 7: e46367, 2012. 
28. Li X, Zhang Z, Yu M, Li L, Du G, Xiao W and Yang H: Involvement of miR-20a in promoting gastric cancer progression by targeting early growth response 2 (EGR2). Int J Mol Sci 14: $16226-16239,2013$.

29. Chai H, Liu M, Tian R, Li X and Tang H: MiR-20a targets BNIP2 and contributes chemotherapeutic resistance in colorectal adenocarcinoma SW480 and SW620 cell lines. Acta Biochim Biophys Sin (Shanghai) 43: 217-225, 2011.

30. Wang ZH, Chen H, Guo HC, Tong HF, Liu JX, Wei WT, Tan W, Ni ZL, Liu HB and Lin SZ: Enhanced antitumor efficacy by the combination of emodin and gemcitabine against human pancreatic cancer cells via downregulation of the expression of XIAP in vitro and in vivo. Int J Oncol 39: 1123-1131, 2011.

31. Li F and Sethi G: Targeting transcription factor NF-kappaB to overcome chemoresistance and radioresistance in cancer therapy. Biochim Biophys Acta 1805: 167-180, 2010.

32. Jono H, Lim JH, Chen LF, Xu H, Trompouki E, Pan ZK, Mosialos G and Li JD: NF-kappaB is essential for induction of CYLD, the negative regulator of NF-kappaB: Evidence for a novel inducible autoregulatory feedback pathway. J Biol Chem 279: 36171-36174, 2004.

33. Regamey A, Hohl D, Liu JW, Roger T, Kogerman P, Toftgard R and Huber M: The tumor suppressor CYLD interacts with TRIP and regulates negatively nuclear factor kappaB activation by tumor necrosis factor. J Exp Med 198: 1959-1964, 2003.
34. Kovalenko A, Chable-Bessia C, Cantarella G, Israël A, Wallach D and Courtois G: The tumour suppressor CYLD negatively regulates NF-kappaB signalling by deubiquitination. Nature 424: 801-805, 2003.

35. Brummelkamp TR, Nijman SM, Dirac AM and Bernards R: Loss of the cylindromatosis tumour suppressor inhibits apoptosis by activating NF-kappaB. Nature 424: 797-801, 2003.

36. Xia JT, Chen LZ, Jian WH, Wang KB, Yang YZ, He WL, He YL, Chen D and Li W: MicroRNA-362 induces cell proliferation and apoptosis resistance in gastric cancer by activation of NF- $\kappa \mathrm{B}$ signaling. J Transl Med 12: 33, 2014.

37. Manu KA, Shanmugam MK, Ramachandran L, Li F, Fong CW, Kumar AP, Tan P and Sethi G: First evidence that $\gamma$-tocotrienol inhibits the growth of human gastric cancer and chemosensitizes it to capecitabine in a xenograft mouse model through the modulation of NF- $\kappa \mathrm{B}$ pathway. Clin Cancer Res 18: 2220-2229, 2012.

38. Vucic D and Fairbrother WJ: The inhibitor of apoptosis proteins as therapeutic targets in cancer. Clin Cancer Res 13: 5995-6000, 2007.

39. Zhou R, Hu G, Gong AY and Chen XM: Binding of NF-kappaB p65 subunit to the promoter elements is involved in LPS-induced transactivation of miRNA genes in human biliary epithelial cells. Nucleic Acids Res 38: 3222-3232, 2010. 